\title{
Editorial
}

\section{C-Based Design of Heterogeneous Embedded Systems}

\author{
Christoph Grimm, ${ }^{1}$ Axel Jantsch, ${ }^{2}$ Sandeep Shukla, ${ }^{3}$ and Eugenio Villar ${ }^{4}$ \\ ${ }^{1}$ Vienna University of Technology, Vienna 1040, Australia \\ ${ }^{2}$ Royal Institute of Technology, 10044 Stockholm, Sweden \\ ${ }^{3}$ Virginia Tech, Blacksburg, VA 24061, USA \\ ${ }^{4}$ University Cantabria, 39005 Santander, Spain \\ Correspondence should be addressed to Christoph Grimm, grimm@ict.tuwien.ac.at
}

Received 14 July 2008; Accepted 14 July 2008

Copyright (C) 2008 Christoph Grimm et al. This is an open access article distributed under the Creative Commons Attribution License, which permits unrestricted use, distribution, and reproduction in any medium, provided the original work is properly cited.

With the proliferation of all kinds of electronic devices and our increased dependences on electronics in every aspect of our lives from handheld PDAs, cell phones, medical and prosthetic devices to automotives and fly-by-wire airplanes, embedded systems have changed dramatically. Such changes have given rise to increasingly complex applications on resource constrained embedded platforms, and new innovative system architectures. On the application side, embedded systems are not any more only invisible computers that do one dedicated task, but also ones which sense, observe, decide, act, and are networked with their environment (ambient intelligence).

Obviously, the extended role of embedded systems is not without impact on the hardware and system architecture. Embedded architectures have evolved from a low-end processor with application-specific coprocessors to networked multiprocessor systems including sensors, analog/mixedsignal, and RF components, giving rise to a high degree of heterogeneity which in turn requires sophisticated and heterogeneous modeling techniques. Because these systems are within the space of the physical world requiring interaction with analog physical phenomena, and communicate through radio-frequency ( $\mathrm{RF}$ ) communication links, the newer architectures are aptly referred to as "embedded analog/mixed-signal systems" (E-AMSs). This nomenclature captures the new complexity and heterogeneity that arise from the fact that HW/SW systems and analog, mixed-signal, RF blocks are so functionally interwoven.

To handle this growing complexity and heterogeneity, new methods and tools are required that are able to handle such intricate and closely coupled heterogeneity between software, hardware, and other (e.g., analog/RF) components.
Even though the standard hardware description languages such as Verilog and VHDL have been extended over the years to model analog and mixed signal designs, the complexity ensuing from the close interaction of hardware, software, and mixed signal domains, and the multiple models of computation has necessitated newer system level languages and surrounding methodologies. One pragmatic approach championed by researchers and the industry has been to use existing software languages such as $\mathrm{C}$ and $\mathrm{C}++$ for the design of the overall systems due to the dominant role of software. This helps tremendously in hardware/software cosimulation in the early stages of design, and also allows one to dynamically experiment with hardware/software partitioning for better performance. Among these C-based system level languages, SystemC has evolved as an industry standard for design of HW/SW systems - but with limited ability to deal with complex issues that arise when designing E-AMS systems. Hence, more research and development of tools and standards are required in this field.

This special issue on "C-based design of embedded systems" of the EURASIP journal on Embedded Systems includes 10 articles that will certainly give the readers an overview on the ongoing research towards a design technology that integrates all kind of components for the realization of future "ambient intelligence" systems.

The first two articles offer a deeper insight into the problems and also potential solutions: the first paper "Power aware simulation framework for wireless sensor networks and nodes" by Glaser et al. deals with new methods to optimize power consumption in energy self-sufficient sensor networks, based on SystemC and OMNET. The second paper "Modeling field bus communications in mixed signal 


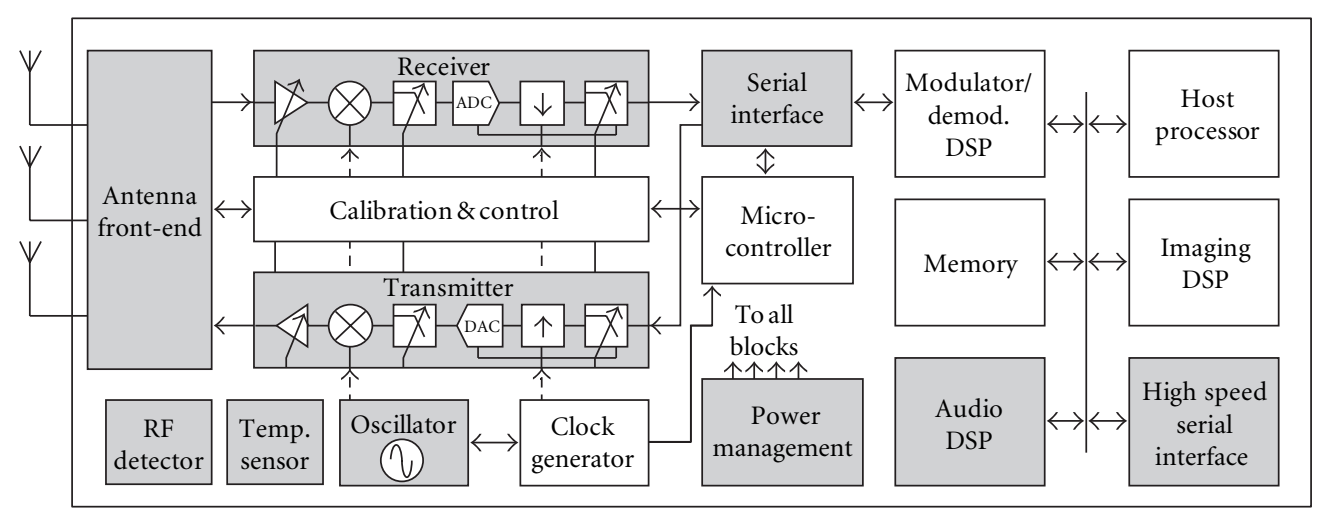

FIGURE 1: Example of an embedded analog/mixed-signal architecture: communication system (from: "An Introduction to Modeling Embedded Analog/Mixed-Signal Systems using SystemC AMS Extensions"; http://www.systemc.org/).

embedded systems" by Denoulet et al. uses the upcoming OSCI SystemC AMS extensions to deal with heterogeneity. Although AMS extensions still are changing, this case study also gives a valuable insight into capabilities of this upcoming standard.

A second group of contributions focuses on new tools and languages. The third paper "System-on-chip environment: A SpecC-based framework for heterogeneous MPSoC design" by Doemer et al. describes a design framework and methodology for heterogeneous MPSoCs based on SpecC, while the fourth paper "Novel methodology for functional modeling and simulation of wireless embedded systems" by Morales et al. addresses modeling and simulation of wireless embedded systems by bringing together the functional models of the baseband algorithms written in C language with the circuit descriptions at behavioral level in Verilog or Verilog-AMS in a single kernel environment, most notably from the viewpoint of the (semiconductor) industry. The fifth paper "Bridging MoCs in SystemC specifications of heterogeneous systems" by Haase et al., also in the context of the upcoming SystemC AMS extensions, describes some means to support the design refinement process targeted by the AMS extensions.

At a higher level, the abstract modeling, IP integration, and synthesis are of crucial importance which are tackled by the sixth paper entitled "Combining UML2 application and SystemC platform modeling for performance evaluation of real-time embedded systems" by Kreku et al., and the seventh paper "Automated integration of dedicated hardwired IP cores in heterogeneous MPSoCs designed with ESPAM" by Nikolov et al., dealing with automated integration of IP cores in heterogeneous MPSoCs. High-level synthesis and its impact on the design of heterogeneous embedded systems are evaluated in the eighth article with the title "Quantitative evaluation of the impact of high-level synthesis on heterogeneous embedded systems design" by Hammami et al.

Last but not least: validation. Validation of E-AMS is may be the most challenging issue. Approaches to improve verification coverage range from model-driven validation in the ninth article "Model-driven validation of System C designs" by Patel et al. to simulators that allow designers to integrate accurate behavior of analog and RF behavior into an overall system simulation in the tenth contribution with the title "System level modeling of RF IC in SystemC-WMS" by Orcioni et al.

We sincerely hope that this special issue will provide the readers with a good understanding of ongoing research and a sense of the challenges on the road ahead.

Christoph Grimm
Axel Jantsch
Sandeep Shukla
Eugenio Villar 